\title{
On the Earth's magnetic field and the Hall effect
}

\author{
J. E. Allen \\ University College, Oxford, OX1 4BH, UK
}

Received: 12 June 2002 - Revised: 14 October 2002 - Accepted: 15 January 2003

\begin{abstract}
In a recent paper de Paor put forward a new theory of the Earth's magnetic field that depended on the Hall effect as an energy transfer mechanism. The purpose of this paper is to demonstrate that the mechanism invoked is unimportant except in certain gaseous plasmas.
\end{abstract}

\section{Introduction}

A new theory of the Earth's magnetic field has been put forward recently that depends on the Hall effect (de Paor, 2001). In this theory the Hall effect is called upon as a mechanism for transferring a flow of electrical energy from one direction to another orthogonal to the first. This is illustrated in Fig. 1, which shows a sample of conducting material placed in a magnetic field; de Paor (loc. cit.) considers that a flow of energy in the $x$-direction is transferred, in part, to the $z$-direction. It is shown in the present paper that, although such a process does take place, the effect is infinitesimally small in solids and liquids. It is extremely unlikely, therefore, that the mechanism is important in the production of the Earth's magnetic field.

\section{The Hall effect}

We shall start by presenting a simple theory of the Hall effect (Kittel, 1996; Solymar and Walsh, 1997). Suppose that we have a uniform electric field $E_{x}$ in the $x$-direction and a magnetic field $B$ in the $y$-direction. The electrons drifting in the $x$-direction will experience a Lorentz force in the $z$ direction. Let us denote the electron drift velocities in the $y$ and $z$-directions by $u, v$, respectively. The mean force in the $x$-direction will be $(-e) u B$ and the mean force in the $x$ direction will be $(-e)\left(E_{x}-v B\right)$. In this notation $e$ is the magnitude of the electronic charge. Thus we obtain the fol-

Correspondence to: J. E. Allen (john.allen@univ.ox.ac.uk)

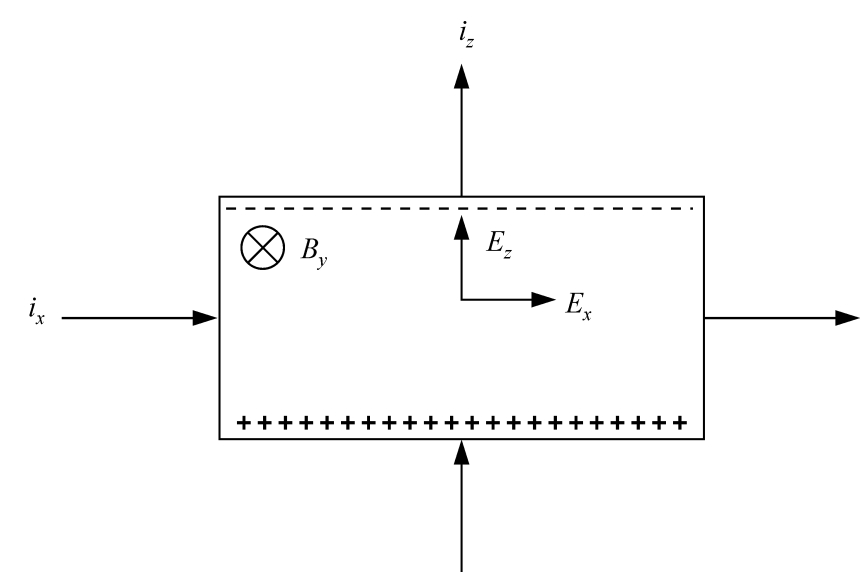

Fig. 1. To illustrate the Hall effect, the charge carriers are assumed to be electrons, which flow in the positive $z$-direction (in the Hall circuit).

lowing momentum balance equations, for the two directions.

and

$$
\begin{aligned}
& (-e)\left(E_{x}-v B\right)=m u v_{c} \\
& (-e) u B=m v v_{c}
\end{aligned}
$$

where $v$ is the collision frequency. From the second equation we have

or

$$
v=-\left(\frac{e B}{m}\right) \tau u, \quad \text { where } \quad \tau=1 / \nu_{c}
$$

$$
v=-(\omega \tau) u
$$

where $\omega$ is the electron gyrofrequency $(e B / m)$. Substitution in the first equation gives

or

$$
\begin{aligned}
& u=-\frac{1}{1+(\omega \tau)^{2}} \frac{e \tau}{m} E_{x} \\
& u=-\frac{1}{1+(\omega \tau)^{2}} \mu E_{x}
\end{aligned}
$$




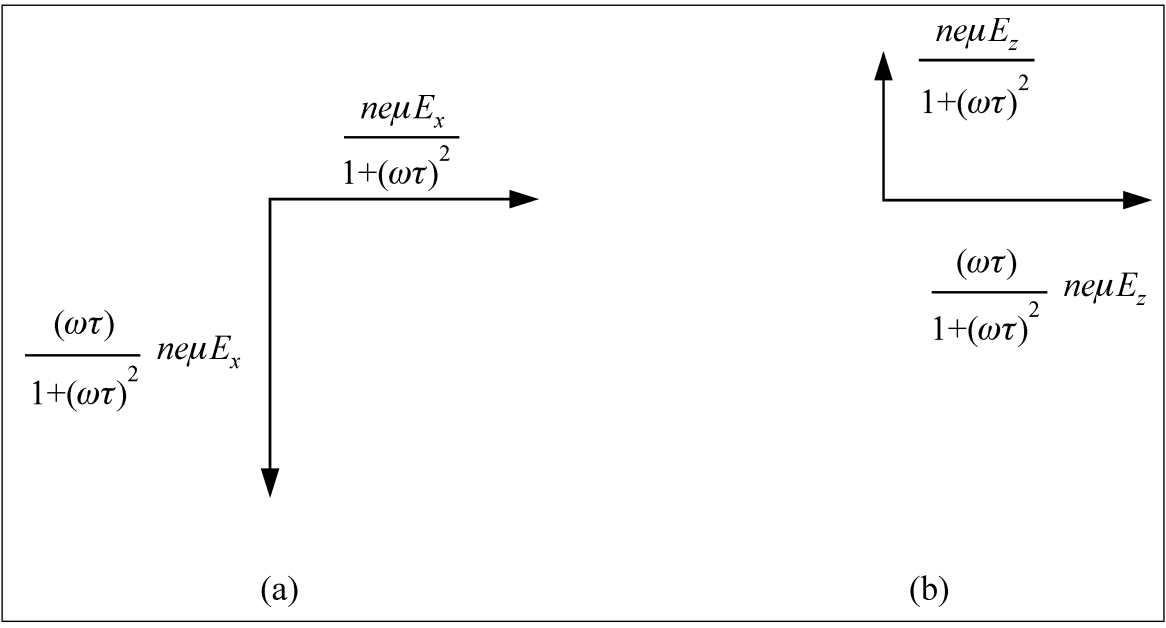

Fig. 2. (a) the current densities due to $E_{x}$ and $B_{y},(\mathbf{b})$ the current densities due to $E_{z}$ and $B_{y}$. On open circuit $E_{z}=$ $(\omega \tau) E_{x}$; at maximum power output in the Hall circuit $E_{z}=(\omega \tau) E_{x} / 2$. where $\mu$ is the mobility. The quantity $(\omega \tau)$ is known as the Hall parameter and the expression $\mu /\left(1+(\omega \tau)^{2}\right)$ is sometimes referred to as "the cross-mobility".

In addition we have

$v=\frac{\omega \tau}{1+(\omega \tau)^{2}} \mu E_{x}$

The current in the $z$-direction is known as the Hall current. The resulting current densities are given by the following two equations, where $n$ is the electron density,

$j_{x}=\frac{1}{1+(\omega \tau)^{2}} \mu n e E_{x}$

and $\quad j_{z}=-\frac{(\omega \tau)}{1+(\omega \tau)^{2}} \mu E_{x}$

We must now consider the effect of impedance in the Hall current circuit. The above calculation applies only when there is no electric field in the $z$-direction; in general such a field will be developed as shown in Fig. 1 (c.f. de Paor, 2001). The current density in the $z$-direction due to $E_{z}$ is indicated in Fig. 2; the associated Hall current in the $x$ direction is also shown and augments that due to $E_{x}$. The total current density in the $x$-direction is now

$j_{x}=\frac{n e \mu}{1+(\omega \tau)^{2}}\left[E_{x}+(\omega \tau) E_{z}\right]$

and that in the $z$-direction is

$j_{z}=\frac{n e \mu}{1+(\omega \tau)^{2}}\left[E_{z}-(\omega \tau) E_{x}\right]$

If the current in the $z$-direction is zero, due to a high impedance circuit, $E_{z}=(\omega \tau) E_{x}$ and $j_{x}=n e \mu E_{x}$, i.e. the magnetic field has no effect on the current flow. The electric and magnetic forces in the $z$-direction are balanced.

The input power to the system is given, in general, by

$W_{i}=\frac{n e \mu E_{x}}{1+(\omega \tau)^{2}}\left[E_{x}+(\omega \tau) E_{z}\right]$

where we have considered a unit volume, for convenience. The output power is given by $W_{o}=-j_{z} E_{z}$ because current flows in the negative $z$-direction, the flow of electrons being upwards.

$W_{o}=\frac{n e \mu E_{z}}{1+(\omega \tau)^{2}}\left[(\omega \tau) E_{x}-E_{z}\right]$

For a given field in the $x$-direction, the output power is a maximum when $E_{z}=(\omega \tau) / 2 E_{x}$.

In this case

$W_{o m}=\frac{n e \mu E_{x}^{2}}{1+(\omega \tau)^{2}} \cdot \frac{(\omega \tau)^{2}}{4}$

In a solid or a liquid $(\omega \tau) \ll 1$, so that the maximum power available would be

$W_{o m}=n e \mu E_{x}^{2} \cdot \frac{(\omega \tau)^{2}}{4}$

Under these conditions the input power and dissipation are given by

and

$$
\begin{aligned}
& W_{i m}=n e \mu E_{x}^{2}\left[1-\frac{1}{2}(\omega \tau)^{2}\right] \\
& W_{d m}=n e \mu E_{x}^{2}\left[1-\frac{3}{4}(\omega \tau)^{2}\right]
\end{aligned}
$$

It is seen that $W_{i m}$ and $W_{d m}$ are both equal to $n e \mu E_{x}^{2}$, to a high degree of approximation. It should be noted that the subscripts of $W_{i m}$ and $W_{d m}$ refer to the situation when the output power has its maximum value.

It is of interest to examine the case where $(\omega \tau) \gg 1$, which is possible for a gaseous plasma. In this case we have the following results

$$
\begin{aligned}
& W_{o m}=\frac{n e \mu E_{x}^{2}}{4} \\
& W_{i m}=\frac{n e \mu E_{x}^{2}}{2} \\
& W_{d m}=\frac{n e \mu E_{x}^{2}}{4}
\end{aligned}
$$




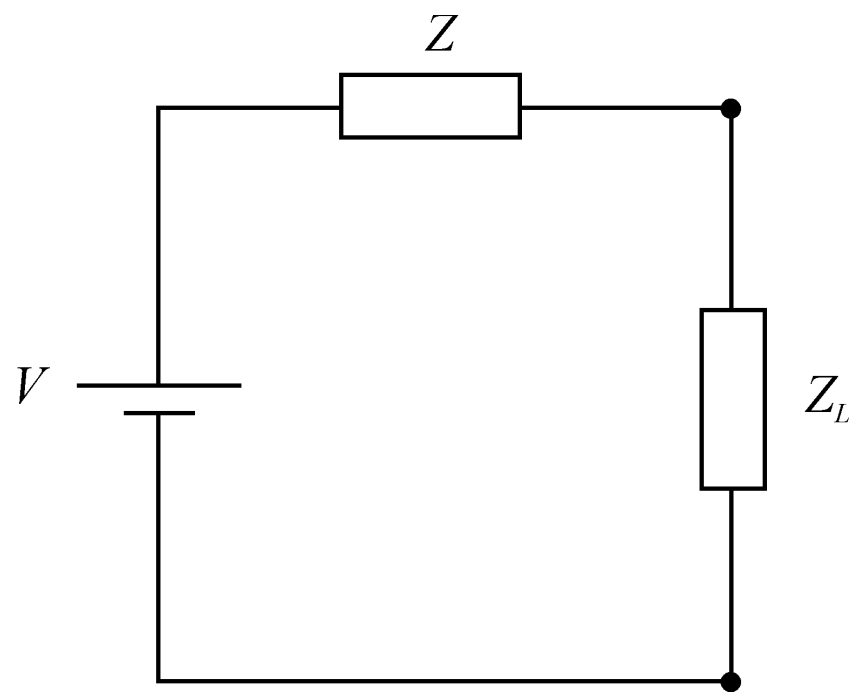

Fig. 3. The equivalent Hall current circuit. $V=(\omega \tau) E_{x} b, Z=$ $\frac{1+(\omega \tau)^{2}}{n e \mu} \cdot \frac{b}{l d}$ and $Z_{L}$ is the load impedance. The dimensions are $b$ along the $z$-axis, $l$ along the $x$-axis and $d$ along the $y$-axis.

It is readily shown from Eqs. (8) and (9) that the power dissipation $\boldsymbol{j} \cdot \boldsymbol{E}$ is given by

$W_{d}=\frac{n e \mu}{1+(\omega \tau)^{2}} E^{2}$

where $E$ is the magnitude of the resultant electric field. The power dissipated in a conductor in the absence of a magnetic field is given by $j^{2} / \sigma$ where $\sigma=n e \mu$. In the present case it can be shown that $j^{2} / \sigma$ is given by the above expression for $W_{d}$. It is seen that the power dissipation, for a given current density, does not depend on the magnetic field. This is a reflection, in macroscopic terms, of the fact that a static magnetic field cannot change the energy of an electron, but only its direction of motion.

\section{Equivalent circuit}

Equation (9) can be rewritten in the form

$E_{z}=(\omega \tau) E_{x}-j_{0}\left[\frac{1+(\omega \tau)^{2}}{n e \mu}\right]$

where $j_{0}=-j_{z}$ and is a positive quantity if $E_{x}>0$. The open-circuit voltage, considering the output circuit, i.e. that associated with current flow in the $z$-direction, is given by

$E_{z o c} b=(\omega \tau) E_{x} b$

and the short-circuit current is given by

$j_{z o c} l d=\frac{\omega \tau}{1+(\omega \tau)^{2}} n e \mu E_{x} l d$

An equivalent circuit can therefore be employed, as illustrated in Fig. 3, in which the internal impedance is given by the ratio of the above two quantities, so that

$Z=\frac{1+(\omega \tau)^{2}}{n e \mu} \cdot \frac{b}{l d}$

The maximum power that can be extracted, given by Eq. (12), is obtained when the external impedance is equal to this quantity. Under these conditions the input power is readily shown to be

(bld) $W_{i m}=\frac{n e \mu b l d}{1+(\omega \tau)^{2}}\left[1+\frac{(\omega \tau)^{2}}{2}\right] E_{x}^{2}$

and the dissipation is given by

(bld) $W_{d m}=\frac{n e \mu b l d}{1+(\omega \tau)^{2}}\left[1+\frac{(\omega \tau)^{2}}{4}\right] E_{x}^{2}$

\section{Comparison with the work of de Paor}

The Hall field, in the $x$-direction, resulting from currents in the $z$-direction is not clearly defined by de Paor. However, the reaction back in the $x$-direction, due to an electric field in the $z$-direction, can be described by the following quantity obtained from Eq. (8)

$E_{x}^{*}=\frac{(\omega \tau)}{1+(\omega \tau)^{2}} E_{z}$

This quantity is itself not an electric field but a measure, in electric field units, $V / m$, of the reaction back in the $x$ direction. It is, in fact, in the positive $x$-direction. We assume that the product of $E^{*}$ and $j_{x}$ is the quantity corresponding to $e_{x} j_{x}$ in de Paor's paper and we shall denote it by $P$, then

$\frac{P}{W_{i}}=\frac{(\omega \tau)}{1+(\omega \tau)^{2}} \cdot \frac{E_{z}}{E_{x}}$

For maximum power extraction $E_{z}=(\omega \tau) / 2 E_{x}$ so that

$\frac{P}{W_{i m}}=\frac{(\omega \tau)^{2}}{2\left[1+(\omega \tau)^{2}\right]}$

If $\omega \tau \ll 1, P / W_{i m}=(\omega \tau)^{2} / 2$. If $\omega \tau \gg 1$, which is possible for a plasma, $P / W_{i m}=1 / 2$ It is of interest to compare the quantity $P$ with the power extracted

$$
\frac{P}{W_{o}}=\frac{(\omega \tau)}{1+(\omega \tau)^{2}}\left[\frac{E_{x}+(\omega \tau) E_{z}}{(\omega \tau) E_{x}-E_{z}}\right]
$$

using Eqs. (8) and (9) for $j_{x}$ and $j_{y}$. It is seen that $P \neq W_{o}$, so that de Paor's assertion (on page 266) is incorrect. At maximum power extraction $E_{z}=(\omega \tau) / 2 E_{x}$, so that

$$
\frac{P_{m}}{W_{o m}}=\frac{2\left[1+\frac{(\omega \tau)^{2}}{2}\right]}{1+(\omega \tau)^{2}}
$$

When $(\omega \tau) \ll 1, P_{m} / W_{o m}=2$, whereas when $(\omega \tau) \gg$ $1, P_{m} / W_{o m}=1$. Thus $P_{m}$ and $W_{\text {om }}$ are of the same order of magnitude, but the output power is infinitesimal if $(\omega \tau) \ll 1$. 
We have previously demonstrated that when $(\omega \tau) \gg 1$, which is possible in a gaseous plasma,

$$
W_{\text {om }}=\frac{n e \mu E_{x}^{2}}{4}
$$

but when $(\omega \tau) \ll 1$, which is the situation in a solid or a liquid, the maximum output power is given by the much smaller quantity

$$
W_{\text {om }}=\frac{n e \mu E_{x}^{2}}{4}(\omega \tau)^{2}
$$

\section{Discussion}

An estimate of the Hall parameter in the Earth's core is required for the purposes of the present note. The magnitude of the magnetic field has been calculated to be $10^{-2}$ tesla or 100 gauss (Hide, 1955; Hide and Roberts, 1979). The resistivity of liquid steel is $\rho=1.6 \times 10^{-6} \mathrm{ohm} \mathrm{m}$, at $1550^{\circ} \mathrm{C}$ (Marr, 1982); the electron density of iron at room temperature is $n=8.5 \times 10^{28} \mathrm{~m}^{-3}$ (Kittel, loc.cit.). Using these figures for an estimate, the Hall parameter is found to be $(\omega \tau)=4.7 \times 10^{-7}$. Data for the Earth's liquid core will be somewhat different from those quoted above. Using this value of $(\omega \tau)$ in the above analysis, however, the maximum fraction of the input power transferred to the Hall circuit is found to be $5.5 \times 10^{-14}$. It is clear that a more accurate figure is not necessary, the effect has been shown to quite negligible.

A simple theory of the Hall effect has been employed in the present paper, but is thought to suffice for the present purpose. The conclusion is that the mechanism of energy transfer proposed by de Paor is unlikely to be of importance in the generation of the Earth's magnetic field; the effect being infinitesimally small. The Sun consists of plasma and the Hall effect may well play a role in sunspots, that question is not pursued here. Electrons have been taken to be the charge carriers, rather than positive holes as in de Paor's paper. If positive charge carriers were present instead, the Hall current would flow in the opposite direction, but the argument presented in this paper would be unchanged. If charge carriers of both signs were present the Hall effect would be reduced in magnitude.
In solids and liquids, at ordinary temperatures and pressures, the Hall effect takes on a different nature. Negligible currents flow in the Hall direction, but the resulting electric field in the Hall direction is of great importance. Its role is to transmit the $\boldsymbol{j} \times \boldsymbol{B}$ force to the bulk of the material (Shercliff 1965), and its effect on the current flow is insignificant. A different situation is that of neutron stars, where the strongest magnetic fields in the universe are found. Some radio and X-ray pulsars have fields of the order of $10^{8}$ tesla $\left(10^{12}\right.$ gauss $)$, these are "young pulsars" which have an age of about $10^{7}$ years. The Hall parameter can reach values of $10^{2}-10^{3}$ in such neutron stars. Studies of the Hall effect in this astrophysical case are being carried out at present. A recent paper is by Urpin and Shalybkov (1999), where further references may be found.

Acknowledgements. The author wishes to thank Professor Raymond Hide for drawing his attention to this problem. He also recalls interesting discussions on the Hall effect with Peter Thonemann, some years ago.

\section{References}

de Paor, A.: A theory of the Earth's magnetic field and of sunspots, based on a self-excited dynamo incorporating the Hall effect, Nonlinear Processes in Geophysics, 8, 265-279, 2001.

Hide, R.: Free hydromagnetic oscillations of the Earth's core and the theory of the geomagnetic secular variation, Phil. Trans. Roy. Soc. A, 259, 615-647, 1966.

Hide, R. and Roberts, P. H.: How strong is the magnetic field in the Earth's liquid core?, Physics of the Earth and Planetary Interiors, 20, 124-126, 1979.

Kittel, C.: Introduction to Solid State Physics, Wiley (Seventh Edition), 1996.

Marr, H. S.: Electromagnetic stirring in continuous casting of steel, Metallurgical Applications of Magnetohydrodynamics, The Metals Society (London), 143-153, 1982.

Shercliff, J. A.: A Textbook of Magnetohydrodynamics, Pergamon Press, Oxford, p. 30, 1965.

Solymar, L. and Walsh, D.: Electrical Properties of Materials, Oxford (Sixth Edition), 1997.

Urpin, V. and Shalybkov, D.: Magnetohydrodynamic processes in strongly magnetized young neutron stars, Mon. Not. R. Astron. Soc., 304, 451-456, 1999. 